\title{
Criminologie
}

\section{La contrebande des produits du tabac au Canada entre 1985 et 1994 et le cas particulier du Québec}

\section{Marc Alain}

Volume 30, numéro 1, printemps 1997

Criminalités économiques

URI : https://id.erudit.org/iderudit/017397ar

DOI : https://doi.org/10.7202/017397ar

Aller au sommaire du numéro

\section{Éditeur(s)}

Les Presses de l'Université de Montréal

ISSN

0316-0041 (imprimé)

1492-1367 (numérique)

Découvrir la revue

Citer cet article

Alain, M. (1997). La contrebande des produits du tabac au Canada entre 1985 et 1994 et le cas particulier du Québec. Criminologie, 30(1), 53-72.

https://doi.org/10.7202/017397ar
Résumé de l'article

The professional smuggling of mass consumption products develops when demand for a product is not adequately fulfilled by the legitimate market. The difficulties encountered in supplying are, in most contemporary cases, caused by real rarity of the desired product. For other cases, however, the rarity is largely virtual in that government taxes aimed at the product in question lead to increasing the product's price to a prohibitive end. This was the case with cigarettes in Canada between 1985 and 1994. Before both, the federal and provincial, governments decided to drastically decrease cigarette taxes in February 1994, the price for a pack of cigarettes was five to six times higher than the same product in the United States. This article begins with a brief review of the contribution made by economists in regard to contemporary smuggling. Focus will be aimed at common characteristics of the smuggling phenomenon across the world. Elements which are more particular to the Canadian smuggling situation will be identified as well. While the difference in the price of cigarettes between Canada and the United States would seem to be the undeniable driving force behind the development of smuggling activities at the countries ' border, one key question remains unexplained. Why was the volume of contraband unequally distributed across Canada even though the price of cigarettes remained largely consistent throughout all provinces? The level of organization of smuggling networks was much higher in Eastern Canada, and particularly in Quebec, than it was in the western provinces. It is argued that the reasons for this are not only due to price, but to a series of political, historical, and geographical factors which allowed cigarette smugglers to function better in Quebec than in the rest of the country.
Tous droits réservés @ Les Presses de l'Université de Montréal, 1997
Ce document est protégé par la loi sur le droit d'auteur. L’utilisation des services d’Érudit (y compris la reproduction) est assujettie à sa politique d'utilisation que vous pouvez consulter en ligne.

https://apropos.erudit.org/fr/usagers/politique-dutilisation/ 


\section{LA CONTREBANDE DES PRODUITS DU TABAC AU CANADA ENTRE 1985 ET 1994 ET LE CAS PARTICULIER DU QUÉBEC. \\ Marc Alain}

The professional smuggling of mass consumption products develops when demand for a product is not adequately fulfilled by the legitimate market. The difficulties encountered in supplying are, in most contemporary cases, caused by real rarity of the desired product. For other cases, however, the rarity is largely virtual in that government taxes aimed at the product in question lead to increasing the product's price to a prohibitive end. This was the case with cigarettes in Canada between 1985 and 1994. Before both, the federal and provincial, governments decided to drastically decrease cigarette taxes in February 1994, the price for a pack of cigarettes was five to six times higher than the same product in the United States. This article begins with a brief review of the contribution made by economists in regard to contemporary smuggling. Focus will be aimed at common characteristics of the simuggling phenomenon across the world. Elements which are more particular to the Canadian smuggling situation will be identified as well. While the difference in the price of cigarettes between Canada and the United States would seem to be the undeniable driving force behind the development of smuggling activities at the countries' border, one key question remains unexplained. Why was the volume of contraband unequally distributed across Canada even though the price of cigarettes remained largely consistent throughout all provinces? The level of organization of smuggling networks was much higher in Eastern Canada, and particularly in Quebec, than it was in the western provinces. It is argued that the reasons for this are not only due to price, but to a series of political, historical, and geographical factors which allowed cigarette smugglers to function better in Quebec than in the rest of the country.

\section{INTRODUCTION}

Deux disciplines se sont principalement intéressées au phénomène de la contrebande, soit l'histoire et l'économie. Si l'histoire nous a permis de

1. Agent de recherche au Centre international de criminologie comparée, Université de Montréal, C.P. 6128, Succ. Centre-ville, Montréal, Québec H3C 3J7. 
mieux connaître certains contrebandiers devenus chez eux des figures mythiques (Béquet, 1972), le fait est, cependant, qu'elle ne nous permet que très peu de comprendre la dynamique contemporaine de la contrebande professionnelle. L'économie a l'avantage de nous montrer à quel point les activités de contrebande au $\mathrm{Xx}^{\mathrm{e}}$ siècle ont de moins en moins à voir avec les exploits des contrebandiers $\mathrm{d}$ 'antan : ceux-ci ont fait place à des réseaux bien structurés, reliant un ensemble de composantes qui se situent d'un bout à l'autre du spectre de la légalité.

Cette manière de poser la question de la contrebande de produits de consommation de masse ${ }^{2} s^{\prime}$ applique tout à fait au cas du Canada, qui, au cours de la décennie 1985-1994, a vu croître de manière exponentielle les activités entourant la contrebande des produits du tabac. Comme nous avançons ici qu'il y a eu croissance exponentielle des quantités de cigarettes introduites au Canada par les contrebandiers, nous démontrerons en premier lieu la véracité de cette proposition. L'un des principaux avantages dont nous disposons ici par rapport aux cas de contrebande contemporains étudiés dans le monde consiste en ceci que nous sommes en mesure de réaliser une estimation de l'évolution des quantités de cigarettes de contrebande sur la base des statistiques officielles du commerce du tabac au Canada.

Le recours à des données économiques et à une méthode issue de la discipline économique rendent tout à fait compte de ce qui a pu se passer entre 1985 et 1994 sur l'ensemble du territoire canadien : il y a eu une très forte croissance des quantités de cigarettes de contrebande au fur et à mesure que le prix de son concurrent légal connaissait une série de hausses toutes plus importantes les unes que les autres, en particulier entre 1991 et 1992. Mais si le prix des cigarettes ne variait que très peu entre chacune des provinces canadiennes, un découpage par provinces de l'importance du

2. Nous pourrions, à la limite, utiiiser le terme de «produit légal », par opposition au trafic de produits illégaux. Nous pensons cependant que le phénomène de la contrebande des produits du tabac demeure, quant à ses caractéristiques fondamentales, très comparable à ce qui s'est déroulé aux États-Unis pendant la prohibition de l'alcool dans les années 1920 et 1930. Mais l'alcool était à cette époque une substance illégale aux États-Unis. Bien qu'illégal, il n'en reste pas moins que l'alcool était, avant la mise en place de la prohibition de même que pendant la plus grande partie de cette période, un produit consommé à une très large échelle par à peu près toutes les couches de la population, ce qui est beaucoup moins le cas des drogues prohibées, à cette époque comme à la nôtre d'ailleurs. Toujours en ce qui a trait aux drogues prohibées, il semble également que ce type de marché obéisse à un rapport offre-demande (Lewis, Hartnoll. Bryer. Daviaid et Mitcheson. 1985, p. 282) très différent de celui de la contrebande de produits de consommation de masse : dans le premier cas, c'est essentiellement l'offre de nouveaux produits ou dérivés qui stimule la demande, tandis que dans le second, le mécanisme est inversé. 
commerce illégal laisse clairement voir que ces activités ont cependant été inégalement réparties. Les provinces de l'est et particulièrement le Québec ont été bien davantage touchées par la contrebande que les provinces de l'ouest du pays. Bien que des données commerciales puissent très bien rendre compte de la situation pancanadienne, la question des différences interprovinciales se doit d'être résolue d'une autre manière. Deux éléments issus, ceux-là, de Ja réflexion en criminologie vont nous permettre de proposer une hypothèse spécifiquement rattachée aux particularités québécoises. Il s'agit, en premier lieu, de la notion des opportunités optimales (Cook, 1986) pour que des réseaux s'établissent entre contrebandiers et clients du marché noir. Dans le cas de Montréal, la première de ces opportunités a été une courte distance et, surtout, une multitude de voies d'accès entre la base de départ des produits de la contrebande, les États-Unis, et le marché constitué par les fumeurs de Montréal.

Si ce premier facteur a joué un rôle important dans la mise en place des réseaux de contrebande, c'est cependant le second qui va leur assurer croissance et solidification : la présence de personnes en mesure de se servir des textes de lois sur la taxation et sur le contrôle de la frontière selon leur propre interprétation. Tant au Canada qu'aux États-Unis, il n'y a guère que certains peuples autochtones qui soient en mesure de le faire. Et à partir du moment où l'un de ces peuples se trouve justement nanti d'une situation géographique idéale, il est peu étonnant que s'y développent, là mieux qu'ailleurs, les réseaux nécessaires à la contrebande professionnelle.

Comme nous baserons l'essentiel de notre exposé sur des faits à teneur tout d'abord économique, nous amorcerons notre réflexion par un examen sommaire de la contribution des économistes qui se sont penchés sur le phénomène de la contrebande contemporaine. Ce résumé de la littérature nous permettra de dégager quelques constantes tout en nous permettant aussi de montrer quelles semblent être les particularités de la situation canadienne. L'un de ces éléments particuliers est que les consommateurs de cigarettes de contrebande ont agi, tout au long de la période, en parfaite connaissance de cause.

Nous verrons ensuite quelles ont été les quantités de cigarettes de contrebande ayant traversé la frontière entre le Canada et les États-Unis entre 1985 et 1994, quels ont été les grands cycles de cette évolution et, finalement, quels ont été les effets observables de la baisse des taxes décrétée en février 1994, baisse qui va ramener le prix du paquet de cigarettes à son équivalent de 1985. Cette première démonstration sera suivie d'une seconde où nous montrerons comment les provinces canadiennes ont été inégalement touchées par le phénomène et comment ce ne sont plus ici des questions de prix qui peuvent expliquer ces différences. 
Finalement, après cette deuxième partie plus empirique de notre exposé, nous consacrerons l'ensemble de la troisième partie de ce texte au cas particulier du Québec. La contrebande de cigarettes a beaucoup plus marqué le Québec que les autres provinces canadiennes en raison (1) de sa situation géographique et (2) du contentieux juridique et politique persistant avec une nation autochtone dont l'un des territoires est traversé par la frontière canadoaméricaine.

\section{CONTREBANDE ET MARCHÉ NOIR DES PRODUITS DE CONSOMMATION DE MASSE : EFFETS ÉCONOMIQUES ET MESURE DU PHÉNOMÈNE}

Il nous faut remonter à 1764 pour retrouver l'une des premières tentatives d'analyse socio-économique du phénomène de la contrebande. Cesare Beccaria va publier, quelques mois seulement avant la parution de l'essai qui l'a rendu célèbre, Dei delitti e delle pene, un court texte de cinq pages dans le périodique $l l$ Caffé $:$ "Tentativo analitico sui contrabandi. » L'auteur y énonce une première tentative mathématique de l'établissement d'une taxe optimale ${ }^{3}$. Les équations proposées par Beccaria ne vont être redécouvertes qu'en 1973, lorsque Bhagwati et Hansen (1973) proposent leurs propres opinions sur le phénomène. Toutefois, l'approche privilégiée par ces auteurs demeure purement économique, excluant de l'analyse les éléments plus sociologiques et légaux ${ }^{4}$, ce qui annonce en fait les deux écoles de pensée quant à l'analyse économique de la contrebande. La première, délaissant d'emblée l'analyse de cas concrets, s'attarde à la détermination des tarifs de taxation optimaux en situation de compétition parfaite (Bhagwati, 1969) et aux effets théoriques de la contrebande sur le bien-être économique. C'est à la seconde école (Richter, 1966) qu'il revient au contraire d'être allée mesurer sur le

3. «Puisque i'aigèbre n'est qu'une méthode precise et rapide de raisonnement ou d'analyse quantitative, elle n'est pas uniquement applicable à la géométrie ou aux autres sciences mathématiques, mais aussi à l'analyse de toutes choses qui peuvent augmenter ou diminuer, tout ce qui a une relation comparative. Il serait donc plausible, jusqu'à un certain point, pour les sciences politiques d'en faire l'usage, puisque ces sciences traitent de débit et de crédit, d'impôts, etc., bref, de toutes choses qui impliquent un calcul ou une notion de quantité et de quantification. Je dis bien jusqu'à un certain point, puisque les principes politiques dépendent en grande partie de nombreuses volontés individuelles et d'une variété considérable de passions qui ne peuvent être déterminées avec précision. " (Beccaria. 1764 p. 1. C'est nous qui traduisons.)

4. "Nonetheless, Beccaria's analysis introduces a private cost factor, the risk of being caught - natural for a criminologist -, that certainly is important for determining the volume of smuggling and that our analysis does not deal with. »(Bhagwati et Hansen. 1973, p. 173.) 
terrain des cas réels de contrebande à très large échelle de produits de consommation de masse pour ensuite tenter d'établir quelques constantes du phénomène.

Malgré cette opposition de points de vue, trois éléments font à peu près l'unanimité. Tout d'abord, l'élément moteur des activités de contrebande est toujours la disparité entre le prix d'une denrée dans un marché national et son prix au cours mondial (Macedo, 1987). La disparité peut être réelle (disparité due à la dévaluation d'une monnaie par rapport à la devise étalon utilisée pour fin de commerce international) ou artificiellement créée (surtaxation imposée par un gouvernement afin de protéger une industrie locale ou d'accroître les recettes fiscales). Dans le cas de la contrebande des produits du tabac au Canada, cette mécanique générale s'explique non pas tant par la disparité entre le prix des produits sur les marchés canadien et mondial, que par la disparité des prix tels qu'ils se pratiquent aux États-Unis et ceux de notre marché.

En second lieu, les auteurs s'entendent pour considérer la contrebande contemporaine comme une activité impliquant surtout des compagnies et des firmes d'import-export (Pitt, 1981; Simkin, 1978). Il existe, selon les tenants d'une approche plus purement économique (Norton, 1987 ; Ray, 1982 ; Bhagwati et Srinivasan, 1978 ; Sheikh, 1972), une concurrence entre des compagnies légitimes et des organisations qui se spécialiseraient dans le commerce illégal, tandis que pour les théoriciens qui tentent d'inclure des variables relevant un peu moins des calculs économétriques (Johnson, 1974) - coûts de répression, niveau de volonté politique de refréner l'activité -. il pourra s'agir d'une seule et même entreprise qui se livre aux deux types d'activités en même temps. Si l'on veut bien inclure, de manière hypothétique pour l'instant, les compagnies canadiennes productrices de produits du tabac dans les réseaux de contrebande, il apparaît qu'en fournissant ces réseaux de manière régulière, elles se trouvaient à peu près dans la même position que les firmes d'exportation de caoutchouc indonésiennes décrites par Pitt (1981). Pour Pitt, le volume des échanges illégaux devient fonction, pour une même firme, du volume des échanges légaux : plus les firmes exportent légalement, plus le volume de la contrebande augmente.

Mais de manière un peu paradoxale, la contrebande est finalement considérée comme une activité qui ne touche qu'assez indirectement le consommateur. Il nous faut en outre reconnaître cette réalité contemporaine des activités de contrebande : elles pallient en général un manque d'approvisionnement en produits légaux (Mshomba, 1992). En conséquence, le consommateur pourrait non seulement ne voir aucune différence de prix entre la denrée légale et celle introduite illégalement mais, mieux encore, il pourrait payer plus cher la denrée introduite via la contrebande - ici, ce sont les 
lois classiques de l'offre et de la demande qui vont établir les prix. Nul doute que la situation canadienne quant aux produits du tabac constitue à cet égard une exception notable : le produit n'a jamais fait défaut et, d'autre part, il demeure que les activités de contrebande se sont très probablement accentuées au fil des hausses successives du prix demandé au consommateur, celuici s'étant trouvé dans la situation où il lui était possible de comparer les prix du produit légal à ceux de son concurrent illégal. Il n'empêche toutefois qu'ici comme dans les cas décrits par les économistes, c'est la demande pour un produit qui en a régi l'offres.

Certains points de dissension ressortent également. Ces éléments tiennent encore une fois essentiellement à l'adhésion à une ou l'autre des deux manières de penser : entre en jeu ici la question des coûts associés à la répression et au contrôle des activités de contrebande. Pour les tenants de la première école, qui excluent ces coûts de leurs analyses, lorsque des conditions très précises sont réunies - équivalence de la monnaie intérieure par rapport aux devises étalons utilisées dans le commerce mondial, capacité limitée d'offre d'un produit pour un marché intérieur - l'existence de la contrebande peut bénéficier à l'économie d'un pays. Les pertes fiscales encourues par les gouvernements se trouveraient en quelque sorte compensées par un surplus de capital disponible chez les consommateurs. Cette conclusion est remise en question par les tenants de l'autre tendance, qui incluent dans leurs calculs les frais associés à la répression de la contrebande. À partir du moment où un gouvernement met en branle des mesures pénales destinées à mettre un frein à la contrebande, il y a évidemment un accroissement des dépenses ; cet accroissement, combiné aux pertes fiscales, ne peut qu'abaisser le seuil de bien-être économique d'un pays. Nul doute que nous ne pourrons qu'adopter cet argument en ce qui concerne la situation canadienne : non seulement les pertes fiscales se sont-elles accrues d'année en añnée, mais la mise sur pied d'escouades policières exclusivement attachées au problème a entraîné des coûts additionnels qui ont fait baisser le seuil de bien-être économique.

5. Nous nous devons de reconnaître qu'au cours du dernier tiers de l'épisode de la contrebande des produits du tabac au Canada, c'est l'offre absolument faramineuse de cigarettes à très bon marché qui a en grande partie conditionné la demande. Cependant, avant ce moment, le peu de différence de prix entre le marché légal et celui du marché noir de même que le l'amateurisme relatif de l'organisation des réseaux de contrebande ont été autant d'éléments qui confirment le mécanisme général de la contrebande des produits de consommation de masse, où c'est la demande qui conditionne l'offre. 


\subsection{Les problèmes de la mesure des quantités de marchandises qui circulent en contrebande.}

Toute mesure des quantités de produits qui circulent en contrebande ne pourra être autre chose qu'un estimé : les «comptes " de la contrebande, surtout à partir du moment où elle devient pratiquement industrielle, ne sont accessibles qu'à des gens ayant tout intérêt à ne rien divulguer. Le chercheur doit donc estimer ces données ja plupart du temps à partir d'indices extérieurs. Le fait demeure toutefois que peu d'auteurs ont réellement tenté la chose, essentiellement pour deux raisons. En premier lieu, tout simplement parce que chaque cas est particulier et que les effets économiques liés à l'activité sont très variables d'un cas à un autre. Des facteurs tels que la situation géographique d'un pays, l'absence ou la présence de concurrence intra- ou extraterritoriale, le type de denrées échangées en contrebande sont autant d'éléments qui vont rendre chacun des cas étudiés très particulier. Il est donc difficile de parler d'une mécanique générale de la contrebande en ce qui concerne le calcul des quantités impliquées. C'est d'ailleurs la raison qu'invoquent les auteurs qui ne choisissent de considérer la contrebande qu'à l'intérieur d'un modèle totalement théorique.

Un second élément rend assez difficile la réalisation d'un estimé concret, et il tient dans une large mesure au fait que la contrebande affecte beaucoup plus les pays en voie de développement que les pays industrialisés : si les comptes du commerce de ces pays sont parfois difficiles à obtenir, ils sont aussi rarement homogènes sur une période de temps suffisamment longue pour bien estimer l'ampleur du problème. C'est exactement le genre de problèmes auquel se sont heurtés Simkin (1974) et Pitt (1981) lorsqu'ils ont tenté d'évaluer l'importance de la contrebande dans les pays du sud-est asiatique. L'un et l'autre admettent à quel point les données commerciales officielles de ces pays sont trompeuses ${ }^{6}$.

Comme nous allons maintenant le voir, nous n'aurons pas à faire face ici à ce genre de difficultés, bien que la mesure de l'évolution des quantités de tabac ayant circulé en contrebande au Canada entre 1985 et 1994 sera, Jà encore, un estimé.

6. "More important, the problem, by its very nature, is statiscically obscure, so that attemps at quantitative estimation are necessarily hazardous. Complete neglect, however, is much worse; it implies a zero estimate which, in the circumstances, would be absurd." (Simkin, 1974, pp. 157-158.) 


\section{L'ÉVOLUTION DES QUANTITÉS DE TABAC AYANT CIRCULÉ EN CONTREBANDE AU CANADA ENTRE JANVIER 1985 ET OCTOBRE 1994}

Contrairement aux situations évoquées plus haut, nous disposons au Canada de données commerciales précises et homogènes pour tout ce qui a trait au commerce des produits du tabac. Statistique Canada tient en effet un compte précis de la production, des ventes intérieures et des exportations sur une base constante depuis 1961 . Si une première analyse de ces tendances entre 1974 et la fin de l'année 1984 montre une stabilité quasi parfaite, il en va tout autrement pour la décennie suivante. C'est à partir du mois de janvier 1985 que les exportations amorcent une croissance lente mais régulière, cependant que l'on assiste à une décroissance équivalente du volume des ventes intérieures.

Ces données concernant l'exportation méritent que l'on s'y arrête quelque peu. En effet, les membres du lobby anti-tabagisme n'ont jamais manqué, tout au long de cette seconde période, d'accuser l'industrie du tabac de se livrer à une politique d'exportation vers les États-Unis, politique sciemment orchestrée afin d'alimenter les réseaux de contrebandiers?. Mais il importe de préciser à ce sujet que le total du volume des exportations ne peut constituer le total du volume de la contrebande. Deux marchés légitimes potentiels existent pour les cigarettes exportées aux États-Unis : celui d'éventuels consommateurs habitant ce pays et qui préféreraient ce type de cigarette, et, surtout, le marché des boutiques hors-taxes. C'est ce second marché qui est de très loin le plus important, le premier étant à toutes fins utiles, négligeable. Ainsi, pendant la période de référence de 1975 à 1984, la quasitotalité de la production exportée sert justement à alimenter ce marché (Conseil canadien des fabricants des produits du tabac, CCFPT, 1994). La Loi canadienne sur les douanes permet en effet à tout résident canadien ayant séjourné $\mathbf{4 8}$ heures ou plus en debors du territoire de ramener au pays des quantités limitées de certaines denrées sans avoir à acquitter les taxes d'accise prévues ; la quantité autorisée est d'une cartouche de cigarettes - 200 cigarettes - par personne.

Toute évaluation ou estimation du volume de contrebande doit tenir compte de ces réimportations légales. Or, ce n'est pas sans poser quelques problèmes : il ne s'agit pas ici d'une variable constante, car plus le prix des cigarettes augmente au Canada, plus on est susceptible de voir des nonfumeurs se prévaloir de l'exemption de taxes, soit pour des amis, soit encore

7. «Quoique l'industrie du tabac se dise opposée à la contrebande, ses activités constituent la genèse du problème. » Société canadienne du cancer, mémoire présenté en janvier 1994 , p. III. 
pour revendre les produits ainsi rapportés. $\mathrm{Si}$, donc, les quantités ramenées légalement sont influencées par l'évolution des prix au Canada, elles le sont également par le nombre de personnes qui traversent la frontière. Comme les données colligées par Statistique Canada ne disent rien du temps que ces personnes ont passé aux États-Unis (c'est-à-dire plus ou moins 48 heures) ni ne mentionnent à quel endroit le passage a été constaté (ce sont des données nationales), nous ne pourrons que recourir à l'évolution des prix pour estimer les quantités de cigarettes réimportées légalement par des résidents canadiens. Nous estimons ces quantités mois après mois à l'aide d'une équation de regression simple :

Équation 1 :

$$
X_{\mathrm{q}}=B_{\theta}+B^{*} \text { PRIX }
$$

Où,

$X_{q}$ : quantité estimée de cigarettes ramenées légalement au Canada ;

$B_{\theta}$ : la constante, ou valeur à l'intercept ;

$B$ : le paramètre $B$ de la variable indépendante, PRIX $^{8}$.

Comme les deux évaluations les plus sérieuses quant à l'évolution du volume de cigarettes de contrebande, celle de Lindquist et coll. (1992, 1993) et celle du Ministère du Revenu (1993), tiennent compte de ces réimportations légales, nous sommes en mesure de comparer nos propres résultats à ceuxci, au tableau suivant.

\section{Tableau 1}

Comparaison de trois estimations du volume de cigarettes réimportées légalement par des résidents canadiens, entre 1984 et 1990 (en millions de cigarettes)

\begin{tabular}{lccc}
\hline Années & $\begin{array}{c}\text { Estimé de } \\
\text { Lindquist et coll. } \\
\text { (1993, p. 19) }\end{array}$ & $\begin{array}{c}\text { Estimé du } \\
\text { ministère du Revenu } \\
\text { (Canada, 1993 : annexe 2) }\end{array}$ & $\begin{array}{c}\text { Estimé obtenu par équation } \\
\text { de régression (à partir de } \\
\text { l'équation n.1) }\end{array}$ \\
\hline 1984 & 71.1 & n.d. & 65,3 \\
1985 & 68,2 & n.d. & 66.8 \\
1986 & 62,8 & n.d. & 72,4 \\
1987 & 71,6 & 107,6 & 75,7 \\
1988 & 80.8 & 162,8 & 78,3 \\
1989 & 85.1 & 186,5 & 82,1 \\
1990 & 91,8 & 175,2 & 90.8 \\
\hline
\end{tabular}

n.d. : non disponible.

8. Le coefficient $R 2$ ajusté de l'équation est $R 2=.75$. Le coefficient Beta de la variable dépendante est de $B$ ta $=.865$, la statistique $F=14,89$ à $\mathrm{p}=0,012$. 


\subsection{Exportations et marché noir.}

Bien qu'il soit plus que probable que, d'année en année, le nombre de non-fumeurs ramenant des cigarettes exemptes de taxes soit allé en augmentant, nous n'avons aucun moyen d'estimer ce phénomène. Mais comme nos résultats sont tout de même assez proches de l'un des deux estimés, nous utiliserons ces données pour calculer le volume de marchandise disponible pour le marché noir en soustrayant notre estimé du volume réimporté légalement du volume total de cigarettes exportées. Avant de présenter cette estimation, voyons comment les données de l'exportation se combinent à celles des réimportations légales.

\section{Graphique 1}

Note : l'ordonné de l'estimation des réimportations légales est à gauche du graphique. tandis que celui des exportations est à droite.

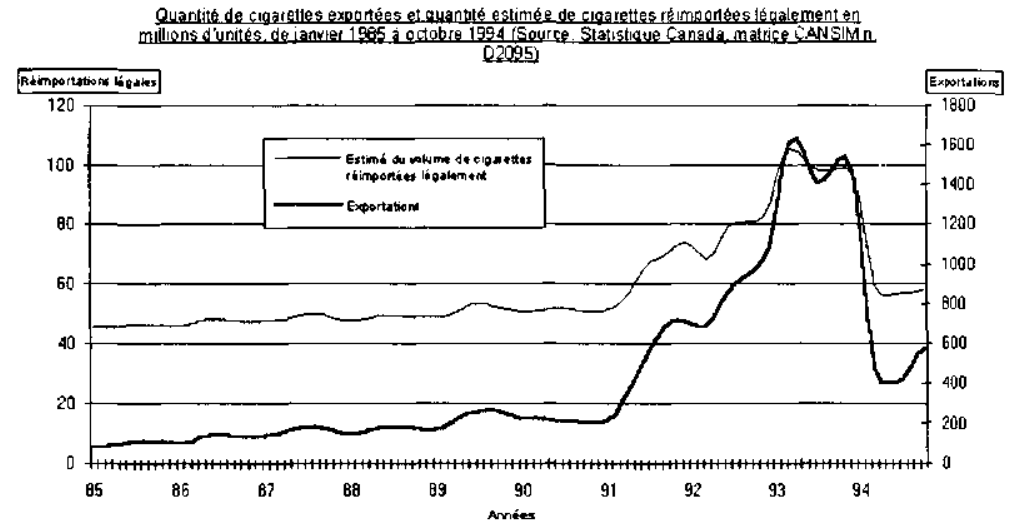

I a courbe de l'estimé du volume de cigarettes réimportées légalement suit les mêmes tendances que celle des exportations. Cependant, peu importe la période considérée, il est clair que le rythme de croissance des exportations est toujours plus accentué que celui des réimportations légales. En un sens, et bien que notre estimation des réimportations n'en tienne pas compte, il est possible que nous observions là le fait qu'au contraire des exportations qui peuvent croître sans réelles limites de volume - il n'y a qu'à affréter plus de camions - le nombre de personnes qui vont traverser la frontière pour plus de 48 heures ne peut pas aller en croissant constamment. Il est donc très possible que notre estimation, en particulier à partir de 1991, soit quelque peu gonflée par rapport à la réalité. Mais de toute manière, comme ces réimportations légales ne constituent finalement qu'une proportion peu 
importante du total des exportations $-6.5 \%$ du total des exportations en mars-avril 1993, au plus fort des exportations - nous pourrons raisonnablement estimer le volume potentiel de cigarettes disponibles sur le marché noir en soustrayant le volume des réimportations légales au volume des exportations.

\section{Graphique 2}

Évolution des quantités de cigarettes offertes sur le marché noir (en tenant compte des réimportations légales) entre janvier 1985 et octobre 1994 en millions de cigarettes

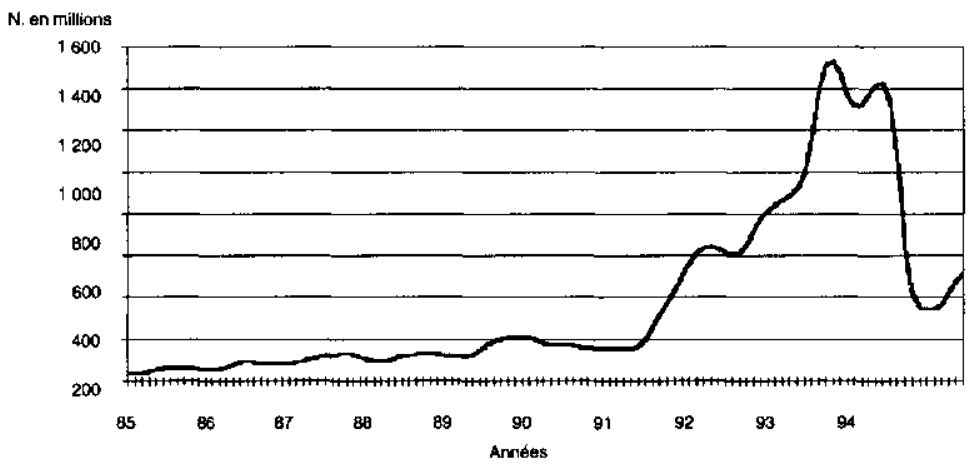

Trois phases distinctes apparaissent dans le temps. De janvier 1985 jusqu'aux premiers mois de 1991, les quantités estimées connaissent une croissance très régulière ; elles vont passer de 75,7 millions d'unités au début de cette première période, à 218,5 millions d'unités en janvier 1990. À ce propos, la forme de la courbe, pour ce qui est de cette première phase, est relativement trompeuse, car, si la croissance n'apparaît pas à ce point importante dans le graphique 2 pour cette première phase, il importe de noter que les quantités vont connaître une croissance annuelle moyenne de $22,5 \%$, avec une pointe maximale de $37,2 \%$ de croissance entre 1986 et 1987.

C'est à partir de 1991 que s'amorce la seconde phase, caractérisée par une véritable explosion des quantités de cigarettes introduites en contrebande : au milieu de 1991, la proportion de cigarettes qui repasse la frontière en contrebande représente $18 \%$ de la production canadienne. Ce pourcentage va atteindre le chiffre assez fantastique de $41,9 \%$ en avril 1993 pour ensuite se stabiliser à plus de $36 \%$ jusqu'en janvier 1994. Pour donner une idée encore plus précise au lecteur de l'ampleur du phénomène, mentionnons que les exportations sont en juin 1991 de 579,1 millions de cigarettes, ce qui représente une augmentation de $160,1 \%$ par rapport au mois de juin 1990 . 
Elles passent ensuite à 895,5 millions d'unités en juin $1992-54,6 \%$ d'augmentation - pour atteindre 2235 millions de cigarettes pendant la pointe de mars 1993 - 149,6\% d'augmentation. Et même au plus fort de cette tendance à l' "exportation », ce ne sont qu'à peine $2,13 \%$ de ces cigarettes qui vont retraverser la frontière en toute légalité.

C'est à partir de février 1994 que la troisième phase de ce développement commence. D'une moyenne mensuelle de 1400 millions de cigarettes, la quantité revient entre 300 et 400 millions d'unités entre mars et juin 1994, une baisse de $75 \%$. Sans contredit, la décision gouvernementale d'abaisser les taxes de près de $70 \%$ en février 1994 n'a pas été sans affecter les quantités de cigarettes disponibles sur le marché noir. Mais là encore, la baisse observée n'est pas vraiment équivalente à ce que la politique des prix de février aurait pu laisser croire : si les prix sont revenus à leur niveau de 1985-1986, les quantités de cigarettes disponibles sur le marché noir ne sont finalement redescendues qu'à celui du milieu de l'année 1991, lors du «boom » des exportations. Non seulement la situation ne s'est-elle pas stabilisée, mais on assiste au contraire à un accroissement, à partir d'août 1994, qui atteint 758,7 millions de cigarettes en octobre, alors que le prix du paquet, lui, ne bouge pas. Sans complètement réussir à mettre de côté l'artefact, il faut recourir à une explication alternative suggérant que d'autres éléments. dont nous n'avons pas tenu compte préalablement, viennent influencer le marché.

Plusieurs provinces canadiennes - Terre-Neuve, la Saskatchewan et la Colombie Britannique - n'avaient pas encore, dans les premiers mois de l'année 1995, abaissé le niveau des taxes sur les produits du tabac. Le problème, cependant, est qu'il n'y a aucune raison pour que des " contrebandiers " — si tant est que de vendre des cigarettes québécoises à des habitants de Terre-Neuve constitue bel et bien une infraction de la Loi sur les douanes et l'accise - se donnent la peine de fournir ces provinces en passant par les États-Unis compte tenu qu'il leur serait beaucoup plus simple de faire passer les cigarettes d'une province à l'autre. En d'autres termes, les mécanismes économiques qui expliquaient, avant la baisse des prix de février 1994, la hausse des exportations et la croissance du volume de marchandises disponibles sur le marché noir ne tiennent plus après ce moment. Comme nous suggérions plus haut que certaines provinces ont été beaucoup plus affectées que d'autres par les activités de contrebande, il devient ici plausible de croire que les réseaux sont devenus à ce point efficaces entre 1985 et 1994 que même la baisse des taxes de février 1994 ne leur a pas complètement enlevé la marge bénéficiaire qui rend l'activité encore attrayante. C'est à partir de cette base de raisonnement que nous pouvons maintenant délaisser les notions économiques pour nous attarder à des éléments qui relèvent davantage de la sociologie et de la criminologie. 


\section{LE CAS PARTICULIER DU QUÉBEC}

Des travaux économiques tout comme de nos propres données empiriques, il ressort que la disparité des prix entre deux marchés serait l'un des principaux facteurs de développement des réseaux de contrebande. Or, avant février 1994, le prix du paquet de cigarettes ne variait pratiquement pas entre les provinces canadiennes; à la limite, cette équivalence aurait pu faire en sorte que la contrebande se soit développée avec une ampleur à peu près similaire partout au Canada. Le fait est, cependant, que si la différence de prix est négligeable entre les provinces canadiennes, ce n'est pas le cas entre chacune de ces provinces et l'État américain limitrophe. comme le montre le tableau 2.

\section{Tableau 2}

Comparaison interprovinciale de la différence du prix d'un paquet de 20 cigarettes entre les provinces et l'Élat américain voisin et niveaux estimés de contrebande en 1993

\begin{tabular}{|c|c|c|c|}
\hline $\begin{array}{l}\text { Provinces } \\
\text { (État américain } \\
\text { limitrophe) }\end{array}$ & $\begin{array}{l}\text { Prix } \\
\text { (en dollars canadiens } \\
\text { pour } 20 \text { cig.) }\end{array}$ & $\begin{array}{l}\text { Différence de prix avec } \\
\text { l'État américain voisin } \\
\text { (en dollars can.)* }\end{array}$ & $\begin{array}{l}\text { Proportion du marché } \\
\text { occupée par la } \\
\text { contrebande** }\end{array}$ \\
\hline Québec (Vermont) & $5,44 \$$ & $3,39 \$$ & $67 \%$ \\
\hline Ontario (New York) & $5,36 \$$ & $1,62 \$$ & $35 \%$ \\
\hline $\begin{array}{l}\text { Colombie-Britannique } \\
\text { (Washington) }\end{array}$ & $5,85 \$$ & $3,55 \$$ & $15 \%$ \\
\hline
\end{tabular}

* Sources : Lindquist et coll., (1993) Lauzon, (1994).

** Sources : Association des détaillants en alimentation, (1994) Gendarmerie royale du Canada, (1994).

Si nous nous en tenions strictement à une hypothèse de différence de prix pour expliquer la variance des activités de contrebande, nous serions en droit de nous attendre à ce que la Colombie-Britannique ait été au moins aussi affectée que le Québec par la contrebande. Il semble pourtant que le Québec a détenu la palme de la plus grande part de marché occupée par les produits de la contrebande, parmi les provinces canadiennes où ces données sont disponibles. Et ici, la particularité québécoise ne peut plus s'expliquer en termes de disparités de prix.

\subsection{Le marché de Montréal et sa proximité avec la frontière canado-américaine : premier déterminant du développement de la contrebande de cigarettes au Québec}

Sur la question du développement des réseaux de contrebande, l'affirmation que, sans consommateurs prêts à acheter des produits de contrebande à bon prix, ces réseaux ne pourront tout simplement pas s'implanter relève 
des lieux communs. Nous avons vu, au cours de la section précédente que, tout bien considéré, les raisons qu'avaient les fumeurs d'acheter des cigarettes de contrebande étaient réparties à peu près également dans les régions de Montréal et de Vancouver. Pourtant, l'analyse de la distribution géographique des activités de contrebande fait clairement ressortir que le Québec en général et, selon les estimations policières de même que selon les deux études de Lindquist et coll.. (1992, 1993), Montréal en particulier ont été beaucoup plus touchés que les autres régions densément peuplées du Canada, notamment les grandes villes canadiennes, telles Toronto ou Vancouver. Si rien ne nous empêche de croire que des réseaux de contrebande auraient pu prendre forme tout le long des quelques cinq mille kilomètres de la frontière canado-américaine, il se trouve cependant que l'infrastructure nécessaire à l'établissement de la contrebande professionnelle n'a pu se faire qu'en un lieu donné où se sont retrouvés en même temps certains éléments conjoncturels favorables.

C'est donc une chose que d'affirmer que sans un bassin de consommateurs suffisamment grand, la contrebande ne peut réellement se développer, mais c'en est une autre que de constater que malgré des marchés à peu près comparables, des villes ont été beaucoup plus touchées par le phénomène que d'autres. A priori, plus la distance entre la frontière américaine et un grand bassin de consommateurs est courte, plus le nombre de voies d'accès est grand, meilleures sont les chances de voir les réseaux se multiplier. C'est exactement ce qui s'est passé à Montréal.

Si Vancouver détient incontestablement la "palme » de la plus courte distance avec la frontière, le problème est ici, d'une part, que les voies d'accès sont limitées (deux voies vraiment pratiques, en fait) et que, $d$ 'autre part, il n'existe pas à proximité de ville américaine importante. Ce qui n'est pas le cas de Toronto, assez proche de Buffalo (115 kilomètres) et de Detroit (300 kilomètres) pour « justifier» un circuit d'exportation. Mais là, un problème de taille se pose aux contrebandiers : la rivière Niagara, qu'il faut traverser pour passer la frontière. Le contrôle frontalier est d'autant plus facile à réaliser que tout le trafic passe par deux ponts.

La conjoncture idéale du nombre de voies d'accès difficiles à surveiller, de la proximité de la frontière américaine et de villes importantes, c'est sans conteste aux alentours de Montréal qu'elle s'est réalisée. Tout d'abord, tous les points de passage de la frontière sont situés sur la terre ferme et ensuite, ils sont beaucoup plus nombreux que dans les cas de Vancouver et de Toronto. Pas moins de six routes importantes traversent la frontière canado-américaine près de Montréal. Qui plus est, comme la plupart de ces points de passage sont situés à la campagne, on peut multiplier par trois le nombre des points de passage si l'on inclut les routes de terre et routes de coupe de bois qui ne 
sont, du côté canadien en tout cas, à peu près pas surveillées. Ces routes, souvent situées en plein bois, se croisent souvent; si les douanes installent un point de contrôle mobile à un endroit précis, les contrebandiers auront tout le loisir d'emprunter un autre point de passage en faisant un léger détour par une autre de ces petites routes. Et, effectivement, au moins à partir de 1990 à la suite de la recrudescence de la surveillance exercée par la GRC autour de l'île de Cornwall durant et après la crise d'Oka (Bonvillain, 1992 ; Hornung, 1991), la presque totalité des cigarettes de contrebande va passer par ces endroits, pour ensuite rejoindre les distributeurs dans les régions de Valleyfield et de Beauharnois.

Une bonne carte routière suffira donc à clarifier la question des opportunités géographiques comme facteur de développement des réseaux de contrebande. Mais ce n'est pas là la seule explication, tant s'en faut. Des Blancs, de plus en plus nombreux et de mieux en mieux organisés, se sont livrés à la contrebande de cigarettes sans s'approvisionner chez les revendeurs et passeurs autochtones. Jamais, toutefois, ces contrebandiers n'ont atteint, en termes de volume de marchandise et d'organisation des réseaux, ce que des Mohawks d'Akwesasne ont réalisé, et ce, à compter du début de la période que nous étudions ici (1986-1987). Pourtant, cette question de distance entre le point d'achat des marchandises de contrebande et le marché où elles sont écoulées ne favorisait quand même pas à ce point les Mohawks, ou en tous cas, certainement pas au point d'amener leur entreprise en situation de quasi-monopole.

D'autres aspects de la situation des Mohawks ont contribué à la réalisation de cette conjoncture: des éléments touchant leur statut juridique de peuple autochtone mais également des éléments qu'il nous faut relier à l'histoire, ancienne et plus contemporaine, des rapports entre Blancs et nations autochtones, entre les gouvernements des uns et les gouvernements des autres.

\subsection{La question Mohawk : droit et histoire}

Deux grands facteurs du contentieux juridique opposant les gouvernements blancs et autochtones sont liés au développement des activités de contrebande chez les Mohawks des trois principales communautés situées au Québec (celle de Kahnawake, au sud de Montréal, celle de Kanesetake, au nord, et celle d'Akwesasne, à environ une heure de route de Montréal). Le premier de ces facteurs est en lien direct avec l'existence de la contrebande : il touche le statut d'Indien et certaines dispositions légales qui les dispensent, à certaines conditions très précises, de payer taxes et impôts. Le second, quant à lui, est en lien nettement plus indirect : il s'agit de l'imposition, par les autorités blanches, d'un mode de gouvernement à l'intérieur d'une communauté qui disposait, bien avant l'arrivée des premiers colonisateurs, d'une 
structure gouvernementale très hiérarchisée, organisée et passablement complexe. Comme nous tenterons de le montrer brièvement ici, c'est en grande partie en raison de l'imposition de cette nouvelle structure gouvernementale que vont apparaître des tensions sociales et des ruptures qui vont permettre à des individus décidés de profiter de ces circonstances conflictuelles pour prendre en main toute une série d'activités en marge de la légalité.

Premier élément en jeu, donc, la question des exemptions de taxes prévues par la loi I-6, dite Loi sur les Indiens. C'est en vertu de l'article 87 de cette loi que tous les Autochtones possédant le statut d'Indien accordé selon les mécanismes prévus par cette même loi peuvent se prévaloir d'une exemption de taxes qui leur est spécifique. Le problème, pour qui tenterait de se prévaloir de cette exemption pour justifier des activités « commerciales " illégales avec les Blancs, serait que cette exemption ne concerne strictement que l'échange de biens entre commerçant autochtone et client autochtone aux seules fins de consommation personnelle. Cette exemption tombe à partir du moment où les biens en question sont, soit vendus à l'extérieur de la réserve, que l'acheteur soit ou non un autochtone, soit vendus à des non-autochtones. La jurisprudence (Hawley, 1994) est absolument univoque à ce sujet : tous les Autochtones qui ont tenté d'invoquer les termes de l'article 87, lors d'activités commerciales qui dépassaient la vente d'un bien à un autre autochtone pour fin de consommation personnelle sur le territoire de la réserve, ont été déboutés en cour. Mais, fait intéressant, l'ont été également les gouvernements provinciaux qui ont tenté d'imposer des taxes spécifiques à la vente des produits du tabac dans les territoires autochtones : l'Ontario à deux reprises, en 1986 et en 1989 ; le NouveauBrunswick, en 1987 (Bélanger, 1988).

$\mathrm{Si}$, en théorie à tout le moins, rien dans les dispositions de la loi I-6 ne permettrait à des Autochtones de se livrer au commerce outre-frontalier sans payer les droits de douanes liés à ces activités, en pratique, toutefois, la situation est beaucoup moins claire à au moins un endroit : Akwesasne. C'est du côté américain de la frontière que le problème prend naissance : ce ne sont pas des Autochtones qui importent, en toute légalité, des cigarettes canadiennes, mais des grossistes ayant un statut particulier, celui d'indian traders, qui sont les seuls à avoir le droit de se livrer à des activités commerciales en gros avec les Mohawks en vertu d'un permis d'exclusivité accordé par le Bureau des Affaires indiennes américain?.

9. Cette pratique tire son origine de l'époque de la conquête de l'ouest du continent américain. En effet, bien peu de commerçants. entre la fin du XIX siècle et le début du $\mathrm{Xx}{ }^{e}$, acceptaient de négliger les petites villes qui naissaient un peu partout pour aller approvisionner les réserves indiennes. On pallia cette carence en offrant à un commerçant 
Si, au Canada, les taxes prélevées sur un paquet de cigarettes sont réparties à parts à peu près égales entre le fisc fédéral et le fisc provincial, aux États-Unis, ce sont les taxes d'État qui sont, et de loin, les plus élevées (cf. le tableau 2). Lorsqu'un grossiste américain importe des cigarettes d'origine canadienne, il n'acquitte que la taxe fédérale (qui est de $4 \%$ ) ; ce sont les détaillants qui vont, par la suite, acquitter la taxe d'État, ce taux variant selon l'État où ils sont établis. Or, les grossistes américains qui font affaires avec les Amérindiens de la Confédération des Six-Nations ont, en plus du statut commercial de grossiste, en tant qu'Indian Traders, l'avantage de pouvoir vendre des produits aux Amérindiens sans que ceux-ci aient à débourser la taxe d'État - l'État de New York ${ }^{10}$, dans ce cas-ci.

Ceci dit, et bien que la législature de l'État de New York conteste que les Mohawks jouissent d'exemptions de taxes propres, il n'en reste pas moins que, jusque-là, leurs activités commerciales sont somme toute légales : la taxe fédérale a été acquittée et les grossistes qui ont importé des cigarettes du Canada les revendent tout simplement aux Mohawks qui, par hypothèse, pourraient bien en être les seuls consommateurs... Ce n'est évidemment pas le cas : les caisses de cigarettes achetées des Indian Traders vont bientôt se retrouver entassées dans de vastes entrepôts situés du côté américain de la réserve d'Akwesasne (The Fifth Estate, 11 janvier 1992). Par bateau l'été, par motoneige l'hiver, les caisses vont traverser le bras de rivière séparant la partie américaine d'Akwesasne de sa portion canadienne, que ce soit du côté du Québec ou du coté ontarien, sur l'île de Cornwall.

C'est ici qu'entrent véritablement en jeu les contrebandiers, des personnes organisées et structurées pour une maximisation des profits tirés de leurs activités. Et c'est en tenant compte d'une série de divisions internes, au sein de ja communauté d'Akwesasne, qu'il faut comprendre l'avènement de ces contrebandiers professionnels. Ces divisions internes ne naissent pas de tensions strictement intérieures de la communauté mohawk, mais de l'imposition à la fois par les autorités américaines et par les autorités canadiennes de conseils de bande, reconnus par ces deux autorités comme seuls

l'exclusivité des échanges commerciaux avec les habitants d'une réserve tout en s'assurant d'au moins un minimum de contrôle quant aux marchandises vendues - la vente d'alccol y étant en particulier interdite - et à leur qualité (cf. Bruchey Struart, 1965. The Roots of American Economic Growth, New York, Harper and Row).

10. L'État de New York conteste cette interprétation du statut autochtone, mais un peu comme c'est le cas au Canada, les protestations d'Albany sont jugées hors-contextes par les Mohawks, qui ne se sentent liés que vis-à-vis de Washington (Akwesasne Notes, 1986, vol. 10, $\mathrm{n}^{\circ} 2$, pp. 2-7). 
interlocuteurs valables (Vachon, 1991"). L'imposition d'un palier supplémentaire de gouvernement dans une communauté qui disposait depuis fort longtemps déjà d'une forme de gouvernement adaptée à sa propre réalité sociale et culturelle a engendré de profondes fractures dans un tissu social déjà très fragilisé par la quasi-impossibilité de maintenir intactes coutumes et activités traditionnelles.

C'est de la division entre les tenants d'un gouvernement traditionaliste et ceux du conseil de bande que va véritablement s'imposer, entre 1979 et 1980, la Mohawk Sovereign Security Force, mieux connue dans le grand public sous l'appellation de Warriors. Bien que la consolidation de cette troisième faction soit considérée avec beaucoup de réticence par chacune des deux autres, les Warriors vont voir leur pouvoir et leur influence dans la communauté mohawk croître très rapidement. $\mathrm{Si}$, au tout début en tout cas, l'influence des Warriors est limitée, deux éléments vont leur permettre de l'accrôttre très rapidement : un discours au ton traditionaliste et les sommes d'argent de plus en plus importantes qui découlent des activités illégales dans lesquelles ils vont se cantonner toujours plus. Car, l'implication des Warriors dans les activités les plus décriées à Akwesasne ne fait actuellement aucun doute; tant dans le cas des bingos que dans celui de la contrebande, sans l'implication de l'organisation des Warriors, jamais ces activités n'auraient connu le développement que l'on a observé entre le milieu des années 1980 et une partie de la décennie suivante.

La combinaison de cette tension politique, de l'absence pendant un temps d'autorité policière reconnue (suites du siège de Raquette Point par des traditionalistes d'Akwesasne entre 1979 et 1980 ; Vachon, 1993 : pp. 2256) et de la quasi-impossibilité pour des autorités policières blanches de faire quoi que ce soit a eu une répercussion fondamentale sur le développement d'activités criminelles de certains Mohawks : une impunité presque totale. Partant, il demeure peu étonnant que ce soient des Mohawks qui aient mis en place la structure des premiers étages des réseaux de contrebande et ont dès lors été en mesure de profiter de la proximité du vaste marché que constituait Montréal.

11. « Évidemment, le Conseil de la nation mohawk affirme que toutes les réclamations devraient être coordonnées au niveau du Grand Conseil ou de la Confédération des Six-Nations et de ceux qu'ils ont désignés. Pourtant, la Loi sur les Indiens requiert que les négociations se fassent exclusivement au niveau des conseils de bande, qui sont modelés sur des gouvernements occidentaux. Tous ces éléments mis ensemble constituent une prescription pour l'impasse actuelle. » (Vachon, 1991, p. 19.) 


\section{CONCLUSION.}

Nous avons ici fait le tour de quelques-unes des multiples dimensions du phénomène de la contrebande tel qu'il s'est déroulé au Canada entre 1985 et 1994. Incontestablement, la politique canadienne de hausses de la taxe sur les produits du tabac pendant cette période a été un puissant élément de développement de la contrebande, qui va peu à peu passer du statut d'activité d'amateurs à celui d'activité professionnelle. C'est devant ce constat qu'au moins deux gouvernements, celui d'Ottawa et celui de Québec, vont en quelque sorte "baisser les bras » en février 1994, et ramener le prix des cigarettes à un niveau à peu près équivalent à celui du marché noir. Or, le problème ici est tout simplement que ces activités ne se sont pas complètement résorbées et que, de plus, notre propre évaluation indique une recrudescence après seulement quelques mois de stabilité.

Ce constat s'explique en grande partie du fait que les réseaux de contrebande se sont beaucoup plus développés à certains endroits qu'à d'autres. Le cas du Québec fait ici figure d'exemple où nous retrouvons combinés deux éléments de conjoncture idéaux, soit :

- la proximité et l'acheminement facile entre une base d'approvisionnement de marchandises et un gros marché, celui de Montréal

- la présence d'individus puissamment organisés tout à fait en mesure d'opérer dans une impunité quasi totale.

Ceci étant dit, nous n'avons fait qu'effleurer ici ces corollaires de la contrebande que sont le marché noir et la banalisation progressive d'un comportement illégal au sein d'une partie importante de la population. Quand on sait que près de deux fumeurs sur trois (Ministère du Revenu, 1993) à Montréal s'approvisionnaient en marchandises illégalement importées, il faut reconnaître que la banalisation de cette habitude est un autre facteur de développement de réseaux professionnels susceptibles de la soutenir, voire peutêtre un facteur essentiel du développement de la contrebande. C'est donc aux raisons ayant rendu possible cette banalisation du recours au marché noir que nous entendons consacrer un prochain texte.

\section{BIBLIOGRAPHIE}

BECCARIA, Cesare Bonesane (1764), "Tentativo analitico sui contrabandi. ", Il Caffé, vol. I, Brescia.

BÉQUET, Paul (1972), Contrebande et contrebandiers. Presses Universitaires de France, Paris.

BÉLANGER, Andrée (1988). Loi concernant les Indiens (S.R.C. c.J-6), texte français avec jurisprudence et commentaires, Secrétariat aux Affaires autochtones, Québec. 
BHAGWATI, Jagdish et Bent HANSEN (1973), «A Theoretical Analysis of Smuggling ». Quarterly Joumal of Economics, $\mathrm{n}^{\circ}$ 87, pp. 377-389.

BHAGWATI, Jagdish et T. N. SRINIVASAN (1978). Smuggling and Trade Policy, in Bhagwati Jagdish (éd.), Illegal Transactions in International Trade, Amsterdam NorthHolland Pub. Company.

BHAGWATI, Jagdish (1969), Fiscal Policies, the Faking of Foreign Trade Declarations and the Balance of Payments, Trade, Tariffs, and Growth. Cambridge M.I.T. Press.

BONVILlAIN, Nancy (1992), The Mohawk, New York, Chealsea House.

Conseil Canadien des fabricants des produits du tabac (1994), La banalisation des produits du tabac, Mémoire présenté au Comité des communes sur la santé en mai 1994.

COOK, Philip J. (1986), « The Demand and Supply of Criminal Opportunities », in Tonry M. et Morris N. (éd.), Crime and Justice, vol. 7, pp. 1-27.

HAWLEY, Donna Lea (1994), The Annotated Indian Act 1993, Toronto, Carswell.

HORNUNG, Rick (1991), One Nation Under The Gun. Toronto, Stoddart.

LAUZON, Léo-Paul (1994), L'industrie du tabac (1987 à 1993), analyse socio-économique, Département des sciences comptables, Montréal, UQAM.

LEWIS, R., R. HARTNOLL, S. BRYER, E. DAVIAID et M. MITCHESON, (1985), "Scoring Smack-The Illicit Heroin Market in L.ondon, 1980-1983 », British Journal of Addiction, $\mathrm{n}^{\circ}$ 80, pp. 281-290.

LINDQUIST, AVEY, MACDONALD et BASKERVILLE (1992), Evaluation de la contrebande des produits du tabac, Toronto.

LINDQUIST, AVEY, MACDONALD et BASKERVJLLE (1993), Evaluation de la contrebande des produits du tabac, mise à jour, Toronto.

MACEDO, Jorge Braga de (1987), « Currency Inconvertibility, Trade Taxes and Smuggling ». Journal of Development Economics, $\mathrm{n}^{\circ} 27$, pp. 109-125.

CANADA, (1993), Consommation et taxation du tabac, Ministère du Revenu, Ottawa.

MSHOMBA, Richard Elias (1992), The Economics of Parallel Markets for Foreign Exchange and Illegal Trade: A Focus on Tanzania, Urbana, University of Illinois Press.

NORTON, Desmond A. G. (1987), «On the Economic Theory of Smuggling », Economica, $n^{0} 55$, pp. 107-118.

PITT, Mark M. (1981), « Smuggling and price disparity. Joumal of Intemational ». Economics, $\mathrm{n}^{\circ} 11$, pp. 447-458.

RAY, Alok (1978), "Smuggling, import objectives, and optimum tax structure». The Quarterly Journal of Economics, $\mathrm{n}^{\circ}$ 92, pp. 509-14.

RICHTER, H.V. (1966), «Indonesia's Share in the Entrepôt Trade of Malaya and Singapore Prior to Confrontation », Malayan Economic Review, Octobre.

SHEIKH. Munir A. (1972), « Smuggling, production and welfare. Journal of International Economics $», n^{\circ} 4$, pp. 355-364.

SIMKIN, C. G. F. (1978), «Indonesia's Unrecorded Trade », in Bhagwati Jagdish ed. Illegal Transactions in Imternational Trade, North-Holland Pub. Company, Amsterdam.

VACHON, Robert (1991), « La nation mohawk et ses communautés », Inter Culture, vol. 24, $\mathrm{n}^{\circ} 4$.

VACHON, Robert (1993), "La dynamique Mohawk de la paix », Inter Culture, vol. 26, $\mathrm{n}^{\circ} 1$. 